EPJ Web of Conferences 73, 02004 (2014)

DOI: $10.1051 /$ epjconf/20147302004

(C) Owned by the authors, published by EDP Sciences, 2014

\title{
Asymmetries of quark sea in nucleon
}

\author{
Harleen Dahiya ${ }^{\mathrm{a}, \mathrm{b}}$ \\ Department of Physics, Dr. B.R. Ambedkar National Institute of Technology, Jalandhar 144011, India
}

\begin{abstract}
The effects of "quark sea" in determining the flavor structure of the octet baryons have been investigated in the chiral constituent quark model. The chiral constituent quark model is able to qualitatively generate the requisite amount of quark sea and is also known to provide a satisfactory explanation of the proton spin and related issues in the nonperturbative regime. The phenomenological implications of the quark sea asymmetries in the nucleon have been investigated to understand the importance of quark sea at lower values of the Bjorken scaling variable.
\end{abstract}

After the first direct evidence for the point-like constituents in the nucleon, identified as the valence quarks with spin-1/2 in the naive constituent quark model (NQM) [1-3], a lot of experiments have been conducted to probe the structure of the proton in the deep inelastic scattering (DIS) experiments. Surprisingly, the DIS results in the early 80's [4] indicated that the valence quarks of the proton carry only about $30 \%$ of its spin and is referred to as the "proton spin crisis" in the NQM. These results provided the first evidence for the proton being composed of three valence quarks surrounded by an indistinct sea of quark-antiquark pairs (henceforth referred to as the "quark sea"). In the present day, the study of the composition of hadrons can be said to be primarily the study of the quark sea and gluons and is considered as one of the active areas in hadronic physics.

The conventional expectation that the quark sea perhaps can be obtained through the perturbative production of the quark-antiquark pairs by gluons produces nearly equal numbers of $\bar{u}$ and $\bar{d}$. Until early 90's a symmetric sea w.r.t. $\bar{u}$ and $\bar{d}$ was assumed, however, the famous New Muon Collaboration in 1991 [5] established the quark sea asymmetry of the unpolarized quarks in the case of nucleon by measuring $\bar{d}-\bar{u}$ giving first clear evidence for the nonperturbative origin of the quark sea. This was later confirmed by the Drell-Yan experiments [6] which measured a large quark sea asymmetry ratio $\bar{d} / \bar{u}$ reminding us that the study of the quark sea is intrinsically a nonperturbative phenomena and it is still a big challenge to perform these calculations from the first principles of QCD.

The chiral constituent quark model $(\chi \mathrm{CQM})$ [7] can yield an adequate description of the quark sea generation through the chiral fluctuations. The basic idea is based on the possibility that chiral symmetry breaking takes place at a distance scale much smaller than the confinement scale. In this region, the effective degrees of freedom are the valence quarks and the internal Goldstone bosons (GBs) which are coupled to the valence quarks [8-10] allowing a simple and intuitive method to investigate

\footnotetext{
${ }^{a}$ H.D. would like to thank Department of Science and Technology, Government of India, for financial support.

be-mail: dahiyah@nitj.ac.in
}

This is an Open Access article distributed under the terms of the Creative Commons Attribution License 4.0, which permits unrestricted use, distribution, and reproduction in any medium, provided the original work is properly cited. 
the principle features of the hadron structure. In the case of quark distribution functions, the latest developments by the NuSea (E866) [11] and HERMES [12] to determine the variation of the seaantiquark ratio $\bar{d}(x) / \bar{u}(x)$ and the difference $\bar{d}(x)-\bar{u}(x)$ with Bjorken scaling variable $x$ have renewed considerable interest in the quark sea asymmetries.

Recently, there has been substantial theoretical progress to take into account the effects of quark sea in determining the flavor structure of the baryons and the question of sea asymmetry has been investigated by several authors using various phenomenological models [13-19]. However, the inclusion of $x$-dependence has not yet been successfully included in the quark distribution functions. Therefore, pending further experiments, it would be interesting to examine the flavor structure of the octet baryons at low energy, thereby giving vital clues to the nonperturbative effects of QCD.

In the present work we determine the sea quark distribution functions and their asymmetries in the octet baryons by phenomenologically incorporating $x$-dependence in the $\chi \mathrm{CQM}$. The extent of contributions coming from the different sea quarks for the octet baryons can also be compared. To understand the relation of the Bjorken scaling variable and quark sea, it would be significant to study its implications in the region $x<0.3$ which is a relatively clean region to test the quark sea structure as well as to estimate their structure functions and related quantities [20].

The key to understand the "proton spin crisis", in the $\chi \mathrm{CQM}$ formalism [9], is the fluctuation process $q^{ \pm} \rightarrow \mathrm{GB}+q^{\prime \mp} \rightarrow\left(q \bar{q}^{\prime}\right)+q^{\prime \mp}$, where $q \bar{q}^{\prime}+q^{\prime}$ constitute the "quark sea" $[9,10,21]$. The effective Lagrangian describing interaction between quarks and a nonet of GBs, can be expressed as $\mathcal{L}=g_{8} \overline{\mathbf{q}}\left(\Phi+\zeta \frac{\eta^{\prime}}{\sqrt{3}} I\right) \mathbf{q}=g_{8} \overline{\mathbf{q}}\left(\Phi^{\prime}\right) \mathbf{q}$, where $\zeta=g_{1} / g_{8}, g_{1}$ and $g_{8}$ are the coupling constants for the singlet and octet GBs, respectively, $I$ is the $3 \times 3$ identity matrix. In terms of the SU(3) and axial U(1) symmetry breaking parameters, introduced by considering $M_{s}>M_{u, d}, M_{K, \eta}>M_{\pi}$ and $M_{\eta^{\prime}}>M_{K, \eta}$ $[9,10]$, the GB field can be expressed as

$$
\Phi^{\prime}=\left(\begin{array}{ccc}
\frac{\pi^{0}}{\sqrt{2}}+\beta \frac{\eta}{\sqrt{6}}+\zeta \frac{\eta^{\prime}}{\sqrt{3}} & \pi^{+} & \alpha K^{+} \\
\pi^{-} & -\frac{\pi^{0}}{\sqrt{2}}+\beta \frac{\eta}{\sqrt{6}}+\zeta \frac{\eta^{\prime}}{\sqrt{3}} & \alpha K^{0} \\
\alpha K^{-} & \alpha \bar{K}^{0} & -\beta \frac{2 \eta}{\sqrt{6}}+\zeta \frac{\eta^{\prime}}{\sqrt{3}}
\end{array}\right) \quad \text { and } \quad q=\left(\begin{array}{l}
u \\
d \\
s
\end{array}\right) \text {. }
$$

The parameter $a\left(=\left|g_{8}\right|^{2}\right)$ denotes the probability of chiral fluctuation $u(d) \rightarrow d(u)+\pi^{+(-)}$, whereas $\alpha^{2} a, \beta^{2} a$ and $\zeta^{2} a$ respectively denote the probabilities of fluctuations $u(d) \rightarrow s+K^{-(0)}, u(d, s) \rightarrow$ $u(d, s)+\eta$, and $u(d, s) \rightarrow u(d, s)+\eta^{\prime}$. The quark sea content of the baryon can be calculated in $\chi \mathrm{CQM}$ by substituting for every constituent quark $q \rightarrow \sum P_{q} q+|\psi(q)|^{2}$, where $\sum P_{q}$ is the transition probability of the emission of a GB from any of the $q$ quark and $|\psi(q)|^{2}$ is the transition probability of the $q$ quark. The flavor structure for the baryon of the type $B(x x y)$ is expressed as $2 P_{x} x+P_{y} y+$ $2|\psi(x)|^{2}+|\psi(y)|^{2}$ and for the type $B(x y z)$ it is expressed as $P_{x} x+P_{y} y+P_{z} z+|\psi(x)|^{2}+|\psi(y)|^{2}+$ $|\psi(z)|^{2}$, where $x, y, z=u, d, s$.

There are no simple or straightforward rules which could allow incorporation of $x$-dependence in $\chi \mathrm{CQM}$. To this end, instead of using an ab initio approach, we have phenomenologically incorporated the $x$-dependence getting clues from Eichten et al. [8], Isgur [2] et al. and Le Yaouanc et al. [3]. The $x$-dependent sea quark distribution functions can be now expressed as $\bar{u}^{B}(x)=\bar{u}^{B}(1-x)^{10}, \bar{d}^{B}(x)=$ $d^{B}(1-x)^{7}, \bar{s}^{B}(x)=s^{B}(1-x)^{8}$ which together with the valence quark distribution functions give the flavor structure of the baryon as $q^{B}(x)=q_{\text {val }}^{B}(x)+\bar{q}^{B}(x)$, where $q=u, d, s$. Using the sea quark distribution functions, the quark sea asymmetries $\bar{u}(x)-\bar{d}(x)$ and $\bar{d}(x) / \bar{u}(x)$ can also be calculated at different $x$ values.

The $x$-dependence of the structure functions $F_{1}$ and $F_{2}$ can be calculated from

$$
F_{2}^{B}(x)=x \sum_{u, d, s} e_{q}^{2}\left[q^{B}(x)+\bar{q}^{B}(x)\right], \quad F_{1}^{B}(x)=\frac{1}{2 x} F_{2}^{B}(x),
$$


where $e_{q}$ is the charge of the quark $q\left(e_{u}=\frac{2}{3}\right.$ and $\left.e_{d}=e_{s}=-\frac{1}{3}\right)$. In terms of the quark distribution functions, the structure function $F_{2}$ for any baryon can be expressed as

$$
F_{2}^{B}(x)=\frac{4}{9} x\left(u^{B}(x)+\bar{u}^{B}(x)\right)+\frac{1}{9} x\left(d^{B}(x)+\bar{d}^{B}(x)+s^{B}(x)+\bar{s}^{B}(x)\right) .
$$

Several important quantities can be obtained from the structure functions of different isospin multiplets. The Gottfried integral $I_{G}^{p n}$ [22] can be expressed in terms of the sea quarks as follows

$$
I_{G}^{p n}=\int_{0}^{1} \frac{F_{2}^{p}(x)-F_{2}^{n}(x)}{x} d x=\frac{1}{3}+\frac{2}{3} \int_{0}^{1}\left[\bar{u}^{p}(x)-\bar{d}^{p}(x)\right] d x .
$$

The Gottfried integral can similarly be calculated for the case of other octet baryons. It is clear from Eq. (4), the flavor symmetric sea leads to the Gottfried sum rule $I_{G}=\frac{1}{3}$ with $\bar{u}^{B}=\bar{d}^{B}$.

After having detailed the contribution of the quark sea and the various asymmetries in the octet baryons of different quark structure, we now discuss the variation of these quantities with the Bjorken variable $x$. For the numerical calculation of the sea quark distribution functions of the octet baryons, we have used the same set of input parameters as detailed in our earlier calculations [21]. The variation of the sea quark distributions $x \bar{u}(x), x \bar{d}(x)$ and $x \bar{s}(x)$ with the Bjorken scaling variable $x$ for $p(u u d)$, $\Sigma^{+}(u u s), \Sigma^{0}(u d s)$ and $\Xi^{0}(u s s)$ is as follows

$$
\begin{aligned}
& \bar{d}^{p}(x)>\bar{u}^{p}(x)>\bar{s}^{p}(x), \quad \bar{d}^{\Sigma^{+}}(x)>\bar{u}^{\Sigma^{+}}(x) \approx \bar{s}^{\Sigma^{+}}(x), \\
& \bar{d}^{\Sigma^{0}}(x)>\bar{u}^{\Sigma^{0}}(x)>\bar{s}^{\Sigma^{0}}(x), \quad \bar{d}^{\Xi^{0}}(x)>\bar{u}^{\Xi^{0}}(x)>\bar{s}^{\Xi^{0}}(x),
\end{aligned}
$$

showing a clear quark sea asymmetry as observed in the DIS experiments $[5,11,12]$. The sea quarks do not contribute at higher values of $x$ and the contributions should be completely dominated by the valence quarks.

The general aspects of the variation of the magnitudes of the sea quark distribution functions $\bar{u}(x), \bar{d}(x)$ and $\bar{s}(x)$ for the octet baryons are able to explain some of the well known experimentally measurable quantities, for example, $\bar{d}^{B}(x)-\bar{u}^{B}(x), \bar{d}^{B}(x) / \bar{u}^{B}(x)$ and the Gottfried integral. These quantities not only provide important constraint on a model that attempts to describe the origin of the quark sea but also provide a direct determination of the presence of significant amount of quark sea in the low $x$ region. When $x$ is small $\bar{d}^{B}(x)-\bar{u}^{B}(x)$ asymmetries are large implying the dominance of sea quarks in the low $x$ region. In fact, the sea quarks dominate only in the region where $x$ is smaller than 0.3 . At the values $x>0.3, \bar{d}-\bar{u}$ tends to 0 implying that there are no sea quarks in this region. The contribution of the quark sea in the case of $\Sigma^{0}$ is particularly interesting because of its flavor structure which has equal numbers of $u, d$ and $s$ quarks in its valence structure. Unlike the other octet baryons, where the $\bar{d}(x)-\bar{u}(x)$ asymmetry decreases continuously with the $x$ values, the asymmetry in this case first increases and then for values of $x>0.1$ it decreases.

A measurement of the Gottfried integral [5, 11] for the case of nucleon has shown a clear violation of Gottfried sum rule from $\frac{1}{3}$ which can find its explanation in a global quark sea asymmetry $\int_{0}^{1}(\bar{d}(x)-\bar{u}(x)) d x$. Similarly, for the case of $\Sigma^{+}, \Sigma^{0}$, and $\Xi^{0}$, the Gottfried sum rules should read $I_{G}^{\Sigma^{+} \Sigma^{0}}=\frac{1}{3}, I_{G}^{\Sigma^{0} \Sigma^{-}}=\frac{1}{3}$ and $I_{G}^{\Xi^{0} \Xi^{-}}=\frac{1}{3}$ if the quark sea was symmetric. However, due to the $\bar{d}(x)-\bar{u}(x)$ asymmetry in the case of octet baryons, a lower value of the Gottfried integrals is obtained. This phenomenological analysis strongly suggests an important role for the quark sea at low value of $x$. New experiments aimed at measuring the flavor content of the other octet baryons are needed for profound understanding of the nonperturbative properties of QCD.

\section{References}

[1] A. De Rujula, H. Georgi and S.L. Glashow, Phys. Rev. D 12, 147 (1975)

[2] N. Isgur, Phys. Rev. D 59, 034013 (1999) 
[3] A. Le Yaouanc, L. Oliver, O. Pene and J.C. Raynal, Phys. Rev. D 15, 844 (1977)

[4] EMC Collaboration, J. Ashman et al., Phys. Lett. B 206, 364 (1988); Nucl. Phys. B 328, 1 (1989); SMC Collaboration, P. Adams et al., Phys. Rev. D 56, 5330 (1997); E142 Collaboration, P.L. Anthony et al., Phys. Rev. Lett. 71, 959 (1993); E143 Collaboration, K. Abe et al., Phys. Rev. Lett. 75, 391 (1995)

[5] New Muon Collaboration, M. Arneodo et al., Phys. Rev. D 50, R1 (1994)

[6] NA51 Collaboration, A. Baldit et al., Phys. Lett. B 253, 252 (1994)

[7] S. Weinberg, Physica A 96, 327 (1979); A. Manohar and H. Georgi, Nucl. Phys. B 234, 189 (1984)

[8] E.J. Eichten, I. Hinchliffe and C. Quigg, Phys. Rev. D 45, 2269 (1992)

[9] T.P. Cheng and Ling Fong Li, Phys. Rev. Lett. 74, 2872 (1995); T.P. Cheng and Ling Fong Li, Phys. Rev. D 57, 344 (1998)

[10] X. Song, J.S. McCarthy, and H.J. Weber, Phys. Rev. D 55, 2624 (1997); X. Song, Phys. Rev. D 57, 4114 (1998)

[11] E866/NuSea Collaboration, E.A. Hawker et al., Phys. Rev. Lett. 80, 3715 (1998); J.C. Peng et al., Phys. Rev. D 58, 092004 (1998); R.S. Towell et al., Phys. Rev. D 64, 052002 (2001)

[12] HERMES Collaboration, K. Ackerstaff et al., Phys. Rev. Lett. 81, 5519 (1998)

[13] M. Alberg, E.M. Henley, and G.A. Miller, Phys.Lett. B 471, 396 (2000); S. Kumano and M. Miyama, Phys. Rev. D 65, 034012 (2002); Fu-Guang Cao and A. I. Signal, Phys. Rev. D 68, 074002 (2003); F. Huang, R.-G. Xu, B.-Q. Ma, Phys. Lett. B 602, 67 (2004); B. Pasquini, S. Boffi, Nucl. Phys. A 782, 86 (2007)

[14] M. Wakamatsu, Phys. Rev. D 44, R2631 (1991); M. Wakamatsu, Phys. Rev. D 46, 3762 (1992); H. Weigel, Phys. Rev. D 55, 6910 (1997); M. Wakamatsu and T. Kubota, Phys. Rev. D 57, 5755 (1998); M. Wakamatsu, Phys. Rev. D 67, 034005 (2003)

[15] Yong Ding, Rong-Guang Xu, and Bo-Qiang Ma, Phys. Rev. D 71, 094014 (2005); Lijing Shao, Yong-Jun Zhang, and Bo-Qiang Ma, Phys. Lett. B 686, 136 (2010)

[16] L.A. Trevisan, C. Mirez, T. Frederico, and L. Tomio, Eur. Phys. J. C 56, 221 (2008); Yunhua Zhang, Lijing Shao, and, Bo-Qiang Ma, Phys. Lett. B 671, 30 (2009); Yunhua Zhang, Lijing Shao, and Bo-Qiang Ma, Nucl. Phys. A 828, 390 (2009)

[17] A.I. Signal and A.W. Thomas, Phys. Rev. D 40, 2832 (1989)

[18] J. Alwall and G. Ingelman, Phys. Rev. D 71, 094015 (2005)

[19] M. Glück, E. Reya, M.Stratmann, and W. Vogelsang, Phys. Rev. D 53, 4775 (1996); D. de Florian, C.A. Garcia Canal, and R. Sassot, Nucl. Phys. B 470, 195 (1996)

[20] CDHS Collaboration, H. Abramowicz et al., Z. Phys. C 17, 283(1983); Costa et al., Nucl. Phys. B 297, 244 (1988)

[21] H. Dahiya and M. Gupta, Phys. Rev. D 64, 014013 (2001); 67, 074001 (2003); Int. Jol. of Mod. Phys. A, 19 29, 5027 (2004); H. Dahiya, M. Gupta, and J.M.S. Rana, Int. Jol. of Mod. Phys. A, 21, 214255 (2006); H. Dahiya and M. Gupta, Eur. Phys. J. C 52, 571 (2007); H. Dahiya and M. Gupta, Phys. Rev. D 78, 014001 (2008); N. Sharma, H. Dahiya, P.K. Chatley and M. Gupta, Phys. Rev. D 81, 073001 (2010)

[22] K. Gottfried, Phys. Rev. Lett. 18, 1174 (1967) 